ESAIM: PROCEEDINGS AND SURVEYS, November 2014, Vol. 46, p. 86-97

ECIT 2012 - Witold Jarczyk, Daniele Fournier-Prunaret, João Manuel Gonçalves Cabral

\title{
THE EMBEDDING PROBLEM IN ITERATION THEORY
}

\author{
MAREK CEZARY ZDUN ${ }^{1}$
}

\begin{abstract}
This is a survey paper on selected topics concerning the embeddability of given mappings in real flows. Particular attention will be paid to the relationship between the problem of the embeddability and functional equations. Let $X$ be a real manifold and $f: X \rightarrow X$ be a homeomorphism. A family of homeomorphisms $\left\{f^{t}: X \rightarrow X, t \in \mathbb{R}\right\}$ such that $f^{t} \circ f^{s}=f^{t+s}$ for $t, s \in \mathbb{R}$ and $f^{1}=f$ is said to be an embedding of $f$. The embedding is of class $C^{r}$ if for every $x \in X$ the mapping $t \rightarrow f^{t}(x)$ is continuous and all $f^{t}$ are of class $C^{r}$. We concentrate on the cases where $\mathrm{X}$ is an open subset of $\mathbb{R}^{N}$, $X$ is a closed and an open interval, and $X$ is a circle. We discuss the following problems: the existence of embeddings with suitable regularity; the conditions which imply the uniqueness of embeddings; the formulas expressing the above embeddings or their general constructions.
\end{abstract}

Résumé. Cet article est une revue de sujets choisis concernant le plongement des fonctions données dans des flots réels. L'attention particulière est prêtée aux relations entre le problème du plongement et des équations fontionnelles. Soit $X$ une variété réelle et $f: X \rightarrow X$ un homéomorphisme. Une famille de homéomorphismes $\left\{f^{t}: X \rightarrow X, t \in \mathbb{R}\right\}$ telle que $f^{t} \circ f^{s}=f^{t+s}$ pour $t, s \in \mathbb{R}$ et $f^{1}=f$ est appelé une plongée de f. La plongée appartient à la classe de $C^{r}$ si pour tout $x \in X$ la fonction $t \rightarrow f^{t}(x)$ est continue et tout $f^{t}$ appartient à la classe de $C^{r}$. Nous nous concentrons sur les cas dans lesquels $\mathrm{X}$ est un sous-ensemble ouvert de $\mathbb{R}^{N}, \mathrm{X}$ est un intervalle fermé et ouvert et $\mathrm{X}$ est un cercle. Nous discutons les problèmes suivants: l'existence des plongées d'une régularité souhaitée, les conditions qui impliquent que ces plongées sont unique, les formules exprimant les plongées mentionnées ci-dessus ou leurs constructions générales.

\section{INTRODUCTION}

Let $X$ be a real manifold and $f: X \rightarrow X$ be a given continuous injection.

Definition 1. A family of homeomorphisms $\left\{f^{t}: X \rightarrow X, t \in \mathbb{R}\right\}$ such that

$$
f^{t} \circ f^{s}=f^{t+s}, \quad t, s \in \mathbb{R}
$$

is said to be a flow or an iteration group. If, in addition, $f^{1}=f$ then the flow is said to be an embedding of $f$ or an iteration group of $f$.

We will say that the embedding is of class $C^{r}$ (monotonic) if for every $x \in X$ the mapping $t \rightarrow f^{t}(x)$ is continuous (monotonic if $X$ is an interval) and $f^{t}$ are of class $C^{r}$

${ }^{1}$ Institute of Mathematics, Pedagogical University of Kraków, Poland, email: mczdun@up.krakow.pl AMS (2000) Subject Classification: 39B12, 37E10, 37E30, 26A18, 26E25, 54C25, 54H15

Keywords: Iteration groups, flows, embedding, functional equations, set-valued functions

Mots-clés: groupes d'iterations, flots, plongée, équations fontionnelles, applications multivoques

(C) EDP Sciences, SMAI 2014 
Definition 2. A family of homeomorphisms $\left\{f^{t}: X \rightarrow X, t \in \mathbb{R}^{+}\right\}$such that

$$
f^{t} \circ f^{s}=f^{t+s}, \quad t, s \geq 0
$$

and $f^{1}=f$ then it is said to be semi-embedding of $f$.

In the theory of dynamical systems the set $X$ is said to be a phase space. The embeddability of a given mapping in an iteration group or semigroup is the same as an extension of a discrete time dynamical system to continuous time dynamical system without alternating the phase space.

The embeddability of a given mapping in a flow may be interpreted as follows: Let us consider a given deterministic process taking place in time. The dynamical rule of this process describes an iteration group $\left\{f^{t}: t \in \mathbb{R}\right\}$ or semigroup $\left\{f^{t}: t \in \mathbb{R}^{+}\right\}$, where iterative index $t$ is interpreted as a time. If the rule of the transformation of the states in a phase space after time 1 describes a function $f$, then $f^{t}$ we can interpret as the changes of the states after the time $t$.

In this note we concentrate only on flows defined on an open subset of $\mathbb{R}^{N}$, closed and open intervals and the circle. We focus on a problem of the existence and uniqueness of embeddings with suitable regularity of one and more functions. In the case of the uniqueness of embedding we give the formulas expressing it and if there is no uniqueness we present the general constructions of these embeddings.

\section{HiSTORICAL REMARKS}

A common source of the embedding problem and the theory of functional equations is the Schröder functional equation

$$
\varphi(f(x))=s \varphi(x) .
$$

It appeared for the first time in 1871 in a paper of E. Schröder connected with continuous iteration groups. However, a fundamental theorem on the existence and uniqueness of analytic solutions of this equation proved G. Koenigs in 1884. Koenigs was the first who considered the problem of the embeddability for the holomorphic functions. Next in 1958 Szekeres created the whole theory of the embeddings of regular functions with one fixed point in the case of real intervals and regions in the complex plane. This subject was developed among others by L. Berg [2], R. R. Coifman [6], [7], [8], S. Dubic [12], M. K. Fort Jr [13], A. Lundberg [33], M. Kuczma [24], [25], D. Krassowska [22], H. Michel [34], A. Smajdor [35], [36], [37], [14], M. C. Zdun [42].

We refer below briefly the ideas of G. Szekeres in the real case (see [39]). Let $I$ be the interval $[0, a)$ and $f$ fulfil the hypothesis:

(H) $f: I \rightarrow I$ is an increasing homeomorphism of class $C^{2}, f^{\prime} \neq 0, \quad f(0)=0$ and $f(x)<x, x \in(0, a)$.

We have the following results on the existence and uniqueness of embedding.

Theorem 1. $1^{\circ}$ If $0<s:=f^{\prime}(0)<1$, then $f$ has the unique $C^{1}$ embedding $\left\{f^{t}: t \in \mathbb{R}\right\}$. Then

$$
f^{t}(x)=s^{t} x+o(x), x \rightarrow 0, \quad t \in \mathbb{R} .
$$

$2^{\circ}$ If $f^{\prime}(0)=0$ and $f(x)=a x^{\mu}+o(x), x \rightarrow 0$, for an $a \neq 0$ and $a \mu>1$ then $f$ has the unique $C^{1}$ embedding such that

$$
f^{t}(x)=\alpha(t) x^{\mu^{t}}+o\left(x^{\mu^{t}}\right), \quad x \rightarrow 0, t \in \mathbb{R},
$$

for an $\alpha(t)>0$. Then $\alpha(t)=a^{\frac{\mu^{t}-1}{\mu-1}}$ for $t \in \mathbb{R}$.

$3^{\circ}$ If $f^{\prime}(0)=1$ and $f(x)=x+a x^{\mu}+o\left(x^{\mu}\right), x \rightarrow 0$ for an $a \neq 0$ and $a \mu$ then $f$ has the unique $C^{1}$ embedding such that

$$
f^{t}(x)=x+\beta(t) x^{\mu}+o\left(x^{\mu}\right), x \rightarrow 0, t \in \mathbb{R},
$$

$$
\text { for } a \beta(t)<0 \text { for } t>0 \text {. Then } \beta(t)=\text { at for } t \in \mathbb{R} \text {. }
$$


The iterations groups satisfying respectively conditions (2), (3) and (4) are called the regular iteration groups of $f$. In each above case we know the formulas expressing the $C^{1}$ embeddings of a given function $f$.

Theorem 2. $\quad 1^{\circ}$ If $0<f^{\prime}(0)=: s<1$ then $C^{1}$ embedding $\left\{f^{t}: t \in \mathbb{R}\right\}$ of $f$ is given by the formula

$$
f^{t}(x)=\varphi^{-1}\left(s^{t} \varphi(x)\right), \quad t \in \mathbb{R},
$$

where

$$
\varphi(x)=\lim _{n \rightarrow \infty} \frac{f^{n}(x)}{s^{n}}
$$

$2^{\circ}$ If $f^{\prime}(0)=1$ then $C^{1}$ embedding $\left\{f^{t}: t \in \mathbb{R}\right\}$ of $f$ fulfilling (3) is given by the formula

$$
f^{t}(x)=\psi^{-1}(\psi(x)+t), \quad t \in \mathbb{R},
$$

where

$$
\psi(x)=\lim _{n \rightarrow \infty} \frac{f^{n}(x)-f^{n}\left(x_{0}\right)}{f^{n+1}\left(x_{0}\right)-f^{n}\left(x_{0}\right)},
$$

and $x_{0} \in I$ is arbitrary chosen.

$3^{\circ}$ If $f^{\prime}(0)=0$ then $C^{1}$ embedding $\left\{f^{t}: t \in \mathbb{R}\right\}$ of $f$ fulfilling (4) is given by the formula

$$
f^{t}(x)=\gamma^{-1}\left(\gamma(x)^{\mu^{t}}\right), \quad t \in \mathbb{R}
$$

where

$$
\gamma(x)=\lim _{n \rightarrow \infty}\left[f^{n}(x)\right]^{\mu^{-n}} .
$$

Iteration groups (5), (7) and (9), where the functions $\varphi, \psi$ and $\gamma$ are given respectively by the formulas (6), (8) and (10) are said to be the principal iteration groups of $f$. Several authors considered the relations between the regular and principal iterations groups of the given functions (see e.g. G. Szekeres [39], A. Lundberg [33], H. Michel [34], R. R. Coifman [6], [7], [8], A. Smajdor and J. Ger [14], D. Krassowska and M. C. Zdun [22]). If in the above theorems we refuse the assumption of the surjectivity of $f$ then we obtain the same results but for semigroups. Also one can weaken the assumptions on the regularity of the function $f$ (see e.g. [26]).

Let us note that the function $\varphi$ is an injective $C^{1}$ solution of Schröder's equation

$$
\varphi(f(x))=s \varphi(x) .
$$

The function $\psi$ is an injective solution of Abel's equation

$$
\psi(f(x))=\psi(x)+1 .
$$

The function $\gamma$ is an injective solution of Böttcher's equation

$$
\gamma(f(x))=\gamma(x)^{\mu}
$$

It is well known that there exist infinitely many continuous embeddings of homeomorphisms. If a given homeomorphism has only fixed points at the ends of its domain then every continuous embedding expresses by the formula (5), where $\varphi$ is a homeomorphic solution of Schröder's functional equation. This solution depends on an arbitrary function. The same embeddings can be also expressed by formulas (7) and (9) and homeomorphic solutions of Abel's and Böther's equations.

More information on this topic one can find also in books [26], [27], [40] and the survey paper [48].

After this introduction let us pass to selected contemporary results. 


\section{EMBEDDING OF DIFFEOMORPHISMS IN REGULAR ITERATION SEMIGROUPS IN $\mathbb{R}^{N}$}

It is well known that for every nonsingular square matrix $S$ there exists a complex matrix $A$ such that

$$
S=e^{A}=\sum_{n=0}^{\infty} \frac{1}{n !} A^{n} .
$$

The matrix $A$ is called a logarithm of $S$.

Obviously $S^{t}:=e^{t A}, \quad t \in \mathbb{R}$ defines a linear embedding of $S$. Moreover, every continuous linear embedding of $S$ is of the above form (see [16]). However, without any additional assumptions on $S$ logarithm $A$ is a complex matrix and consequently embedding $S^{t}$ is complex. We consider the problem when this group is real. W. J. Culver proved the following theorem.

Theorem 3 ( [10]). A linear mapping $S: \mathbb{R}^{N} \rightarrow \mathbb{R}^{N}$ has a linear real embedding if and only if $S$ is nonsingular and the geometric multiplicity of each real negative eigenvalue $\lambda$ of $S$ is even, that is the dimension of the subspace of eigenvectors $\{x: S x=\lambda x\}$ is even.

Moreover, $S$ has a unique linear embedding if and only if all eigenvalues are real and positive and their geometric multiplicities are equal one.

In the case $N=2$ the linear embeddings of $S$ have a relatively simple intelligible form. To present it let us introduce the following notation.

Let $P=[\mathbf{a}, \mathbf{b}] \in M_{2}(\mathbb{R})$ be a matrix with two linearly independent columns $\mathbf{a}, \mathbf{b} \in \mathbb{R}^{2}$. Put

$$
Q(t):=\left[\begin{array}{cc}
\cos t & -\sin t \\
\sin t & \cos t
\end{array}\right]
$$

Theorem 4 ( [38]). Let $S \in M_{2}(\mathbb{R})$ be a nonsingular matrix.

(i) If $S$ has two distinct real eigenvalues $\lambda_{1}, \lambda_{2}>0$ then $S$ has a unique linear real embedding and

$$
S^{t}=P\left[\begin{array}{cc}
\lambda_{1}^{t} & 0 \\
0 & \lambda_{2}^{t}
\end{array}\right] P^{-1},
$$

where $P \boldsymbol{a}=\lambda_{1} \boldsymbol{a}$ and $P \boldsymbol{b}=\lambda_{2} \boldsymbol{b}$.

(ii) If $S$ has an eigenvalue $\lambda \in \mathbb{C} \backslash \mathbb{R}$ then $S$ has countable many linear real embeddings. These embeddings are given by

$$
S^{t}=|\lambda|^{t} P Q(t(\operatorname{Arg} \lambda+2 k \pi)) P^{-1}, k \in \mathbb{Z},
$$

where $\boldsymbol{a}=\operatorname{Re} \boldsymbol{w}, \boldsymbol{b}=\operatorname{Im} \boldsymbol{w}$ and $A \boldsymbol{w}=\lambda \boldsymbol{w}$.

(iii) If $S$ has a unique eigenvalue $\lambda>0$ and $S \neq \lambda$ id then $S$ has a unique real linear embedding. This embedding is given by the formula

$$
S^{t}=\lambda^{t} P\left[\begin{array}{ll}
1 & \frac{t}{\lambda} \\
0 & 1
\end{array}\right] P^{-1}
$$

where $S \boldsymbol{a}=\lambda \boldsymbol{a}$ and $S \boldsymbol{b}=\lambda \boldsymbol{a}+\boldsymbol{b}$.

(iv) If $S=\lambda i d$ then $S$ has infinitely many linear real embeddings. They are given by the formula

$$
S^{t}=|\lambda|^{t} R Q(k \pi) R^{-1}, k \in \mathbb{Z}
$$

where $R$ is an arbitrary nonsingular matrix, $k$ is odd if $\lambda<0$ and $k$ is even if $\lambda>0$.

If $S$ is linearly embeddable then eigenvalues of $S$ satisfy one of the assumptions from the cases (i), (ii), (iii) or (iv). 
After this introduction we move on to the nonlinear case. Let $r$ be a positive integer, $0 \leq \delta \leq 1$ and $U \subset \mathbb{R}^{N}$ be a neighbourhood of the origin. Denote by $D$ if $f_{\delta}^{r}(U)$ the set of all diffeomorphisms $F: U \rightarrow \mathbb{R}^{N}$ of class $C^{r}$ such that $F(0)=0$ and

$$
d^{(r)} F(x)=d^{(r)} F(0)+O\left(\|x\|^{\delta}\right), \quad\|x\| \rightarrow 0 .
$$

We make the general assumption: and

$\left(A_{r}\right) F \in \operatorname{Diff} f_{\delta}^{r}(U)$ and $\left|\lambda_{1}\right| \leq\left|\lambda_{2}\right| \leq \ldots \leq\left|\lambda_{N}\right|<1$, where $\lambda_{1}, \lambda_{2}, \ldots, \lambda_{N}$ are the eigenvalues of $S:=d F(0)$

$$
\left|\lambda_{N}\right|^{r+\delta}<\left|\lambda_{1}\right|
$$

A polynomial function $\eta_{r}: \mathbb{R}^{N} \rightarrow \mathbb{R}^{N}(r \geq 2)$ such that $\eta_{r}(x)=x+\sum_{k=2}^{r} L_{k}(x)$, where $L_{k}$ are homogeneous polynomials of degree $k$, satisfying the system

$$
d^{(k)}\left(\eta_{r} \circ F-S \eta_{r}\right)(0)=0, \quad k=2,3, \ldots, r,
$$

is said to be a formal solution of the Schröder's equation

$$
\varphi(F(x))=S \varphi(x)
$$

of $r$-th order.

If $F$ has no resonance up to the $r$-order that is, the eigenvalues of $S$ satisfy the condition:

$$
\lambda_{1}^{q_{1}} \ldots \lambda_{N}^{q_{N}} \neq \lambda_{i}, q_{i} \geq 0 \text { for } i \in\{1, \ldots, N\} \text { and } 2 \leq \sum_{j=1}^{N} q_{j} \leq r
$$

then there exists a unique polynomial function $\eta_{r}: \mathbb{R}^{N} \rightarrow \mathbb{R}^{N}$ of order $r$ satisfying system (11).

If $r=2$ and $F$ satisfies $\left(A_{2}\right)$ then $F$ has no resonance of second order and, consequently, (12) has a unique formal solution.

Theorem 5. Let $F$ satisfy hypothesis $\left(A_{r}\right)$ for $r \geq 1$ and if $r \geq 3$ (12) has a formal solution of the $r$-th order. $F$ has a $C^{r}$ semi-embedding in a neighbourhood of the origin if and only if the geometric multiplicity of each eigenvalue of $d F(0)$ which is real and negative is even. A $C^{r}$ semi-embedding is unique if and only if the formal solution of (12) is unique and all eigenvalues are real and positive and their geometric multiplicities are equal one.

Theorem 6 ( [46]). If $F$ satisfies $\left(A_{r}\right)$ for $r \geq 1$ and $\left\{F^{t}: U \rightarrow U: t \geq 0\right\}$ is a $C^{r}$ semi-embedding of $F$ then

$$
F^{t}(x)=\varphi^{-1}\left(S^{t} \varphi(x)\right), \quad t \geq 0
$$

where $\left\{S^{t}, t \in \mathbb{R}\right\}$ is a linear embedding of $S$ and $\varphi$ is given by the formula

$$
\varphi(x):=\lim _{n \rightarrow \infty} S^{-n} \circ \eta_{r} \circ F^{n}(x)
$$

where, for $r=1$ with $\delta>0$ and for $r=2$ with $\delta=0$ we take $\eta_{r}=i d$, otherwise $\eta_{r}$ is a formal solution of $r$-order of (12).

Theorem 7 ([46]). If a surjection $F: U \rightarrow U$ satisfies $\left(A_{2}\right)$ with $\delta=0$ has a linear real semi-embedding $\left\{S^{t}, t \geq 0\right\}$, then there exists a unique $C^{2}$ semi-embedding of $F$ such that $d F^{t}(0)=S^{t}$ and it is given by the formula

$$
F^{t}(x)=\lim _{n \rightarrow \infty} F^{-n}\left(S^{t} F^{n}(x)\right), \quad x \in U, \quad t \geq 0
$$


The assumption of the surjectivity can be weakened as follows: there exists a neighbourhood $V \subset U$ of the origin such that

$$
e^{t A} F^{n}[V] \subset F^{n}[U], \quad t \geq 0, \quad n \in \mathbb{N}
$$

In the case of one-dimensional space the formula

$$
F^{t}(x):=\lim _{n \rightarrow \infty} F^{-n}\left(s^{t} F^{n}(x)\right)
$$

for homeomorphism $F$ mapping $[0, \infty)$ onto itself without fixed points in $(0, \infty)$, differentiable at 0 such that $F^{\prime}(0)=: s>1$ has been extensively investigated by J. Domsta (see [11]). He has shown that:

(1) Every semi-embedding $\left\{F^{t}, t>0\right\}$ of $F$ such that all $F^{t}$ are differentiable at 0 is given by formula (13).

(2) If $F$ is continuous and differentiable at zero with $F^{\prime}(0)>1$, but is not sufficiently regular, then the following singularities may happen: $(i)$ limit (13) does not need to exist; ( $i i)$ limit (3) exists, but (13) does not define a semigroup; (iii) limit (13) exists and defines a semigroup, but $F \neq F^{1}$.

\section{Continuous embedding of homeOMORPhisms of THE Plane}

Definition 3. A homeomorphism of the plane onto itself without fixed points which preserves the orientation is said to be a Brouwer homeomorphism or a free mapping.

The Brouwer homeomoprhisms are always embeddable but only locally. This property shows the following theorem.

Theorem 8 ([3]). Let $f: \mathbb{R}^{2} \rightarrow \mathbb{R}^{2}$ be a Brouwer homeomorphism. Then for every $p \in \mathbb{R}^{2}$ there exist a simply connected neighbourhood $U_{p}$ of $p$ and a $C^{0}$-embedding $\left\{f^{t}: U_{p} \rightarrow U_{p}, t \in \mathbb{R}\right\}$ of $\left.f\right|_{U_{p}}$. Moreover, this embedding is of the form

$$
f^{t}(x)=\varphi^{-1}\left(\varphi(x)+t e_{1}\right), \quad t \in \mathbb{R},
$$

where $e_{1}=(1,0)$ and $\varphi: U_{p} \rightarrow \mathbb{R}^{2}$ is a homeomorphism satisfying Abel's equation

$$
\varphi(f(x))=\varphi(x)+e_{1}
$$

for $x \in U_{p}$.

Equation (15) has infinitely many homeomorphic solutions. They depends on an arbitrary function defined on the closed strip bounded by two disjoint lines $K$ and $f[K]$. For details see [29].

The free mappings need not be embeddable globally on the plane. However, we can determine and characterize the groups of homeomorphisms conjugated to the group of translations. At this purpose we introduce the following definition.

Definition 4. Let $D \subset \mathbb{R}^{2}$ be a simply connected region. A homeomorphism $f$ of $D$ onto itself such that every Jordan domain $B \subset D$ meets at most a finite number of its images $f^{n}[B]$ is said to be a Sperner homeomorphism.

Theorem 9 ( [32]). Every Sperner homeomorphism is $C^{0}$-embeddable. If $\left\{f^{t}: D \rightarrow D, t \in \mathbb{R}^{t}\right.$ is a $C^{0}$ embedding of a Sperner homeomorphism $f: D \rightarrow D$ then it is given by (14) for a homeomorphism $\varphi: D \rightarrow \mathbb{R}^{2}$ satisfying Abel's equation (15). Every Sperner homeomorphism has infinitely many $C^{0}$ embeddings, they depend on an arbitrary function.

If a free mapping $f$ is a diffeomorphism of class $C^{r}$ then $f$ has local $C^{r}$ diffeomorphic embeddings. More precisely, if in Theorems 8 and 9 we assume additionally that $f$ is a $C^{r}$ diffeomophism and we replace in these theorems the word "homeomorphism" by " $C^{r}$ diffeomorphism" then these new statements will also be true.

More information on the embeddability of free mappings one can find in Z. Leśniak's papers [28] and [31]. 


\section{Embedding of HOMEOMORPhisms of THE CiRCle}

In this section we consider the homeomorphisms of the circle without periodic points that is such which have irrational rotation number.

Let $\rho(F) \in[0,1)$ be the rotation number of a homeomorphism $F: S^{1} \rightarrow S^{1}$, where $S^{1}:=\{z \in \mathbb{C}:|z|=1\}$. To explain sense of the rotation number let us recall a well-known result of Poincaré.

Theorem 10 (Poincaré). If a homeomorphism $F: S^{1} \rightarrow S^{1}$ has no periodic points then there exists a unique continuous surjective monotone mapping $\Phi: S^{1} \rightarrow S^{1}$ such that

$$
\Phi(F(z))=e^{2 \pi i \rho(F)} \Phi(z), \quad z \in S^{1}
$$

and $\Phi(1)=1$.

This theorem has also very deep applications to the solving the problem of the embeddability.

Put $\Delta:=\left\{F^{n}(z), n \in \mathbb{Z}\right\}^{d}$. The set $\Delta$ does not depend on $z$ and either $\Delta=S^{1}$ or $\Delta$ is a Cantor set (see e.g $[9])$.

Theorem 11 ( [44], [4]). A homeomorphism $F: S^{1} \rightarrow S^{1}$ without periodic points is $C^{0}$-embeddable if and only if $\Delta=S^{1}$. Then $F$ has a unique $C^{0}$-embedding. If $\left\{F^{t}: t \in \mathbb{R}\right\}$ is a $C^{0}$-embedding of $F$ then

$$
F^{t}(z)=\Phi^{-1}\left(e^{2 \pi i \rho(F) t} \Phi(z)\right), \quad t \in \mathbb{R} .
$$

If $F$ is differentiable and the derivative $F^{\prime}$ is of bounded variation then by Denjoy's theorem $F$ is $C^{0}$ embeddable.

If $F$ has periodic points then we have the following characterization of the embeddability.

Theorem 12 ( [44]). A homeomorphism $F: S^{1} \rightarrow S^{1}$ with periodic points is $C^{0}$-embeddable if and only if $F$ preserve orientation and one of the following cases occurs:

(i) $\emptyset \neq\left\{z \in S^{1}: F(z)=z\right\} \neq S^{1}$.

(ii) There exists a positive integer $m$ such that $F^{m}=i d$.

Then $F$ has infinitely many $C^{0}$ embeddings depending on an arbitrary function.

If $F$ has no periodic points and $\Delta \neq S^{1}$ then $F$ may possess the embeddings but nonmeasurable. K. Ciepliński in [4] gave a necessary and sufficient condition for the embeddability in the nonmeasurable iteration groups. In this case $F$ has infinitely many nonmeasurable embeddings (see also [5]).

Denote by $\mathcal{W}$ the set of all sequences $\left\{a_{n}\right\}$ defined by the following way. Let $\left\{k_{n}\right\}$ be arbitrary sequence such that $k_{n} \in\{0, \ldots, n-1\}$. Take $a_{1}:=1$ and

$$
a_{n !}:=a_{(n-1) !}+(n-1) ! k_{n} .
$$

Define the remaining terms $a_{n}$ as the rest of the division $a_{n}$ ! by $n$.

It is easy to verify that $\left\{a_{n}\right\} \in \mathcal{W}$ if and only if

$$
a_{n} \in\{0, \ldots, n-1\}, a_{k n} \equiv a_{n}(\bmod n), \quad k, n \in \mathbb{N} \backslash\{0\} .
$$

For a sequence $\left\{a_{n}\right\}$ define the sets

$$
A_{s}\left(\left\{a_{n}\right\}\right)=\left\{\exp 2 \pi i \frac{m}{n}\left(s+a_{n}\right), \quad n \in \mathbb{N} \backslash\{0\}, m \in \mathbb{Z}\right\}, s \in \mathbb{R} .
$$

On can show that for every $s \in \mathbb{R},\left\{a_{n}\right\} \in \mathcal{W}$ if and only if there exists a function $c: \mathbb{R} \rightarrow S^{1}$ such that $c(x+y)=c(x) c(y)$ for $x, y \in \mathbb{R}, c(1)=\exp 2 \pi s$ and $\operatorname{Imc}=A_{s}\left(\left\{a_{n}\right\}\right)$ (see [4]). 
Put

$$
K_{F}:=\Phi\left[S^{1} \backslash \Delta\right] .
$$

Theorem 13 ( [4]). Let $\alpha(F) \notin \mathbb{Q}$ and $\Delta \neq S^{1}$. The homeomorphism $F$ is embeddable in an iteration group if and only if there exists a sequence $\left\{a_{n}\right\} \in \mathcal{W}$ such that

$$
K_{F} \cdot A_{\alpha(F)}\left(\left\{a_{n}\right\}\right)=K_{F} .
$$

A more difficult problem is $C^{r}$-embedding of diffeomorphisms. The solution of this problem is a simple consequence of the very hard results of Herman, Yoccoz, Katznelson and Orstain (see [15], [41], [19]). We present here only two of them. It turns out that $C^{1}$ embedding depends on the rotation number.

Theorem 14 (Herman, Yoccoz). Let $F$ be a $C^{r}$ circle diffeomorphism, $r \geq 3$. Suppose that the rotation number $\rho(F)$ satisfies the Diophantine condition

$$
\left|\rho(F)-\frac{p}{q}\right|>\frac{K}{q^{2+\beta}}, \quad \frac{p}{q} \in \mathbb{Q},
$$

where $K$ and $\beta$ are positive constants. If $r>2 \beta+1$ then $F$ has a unique $C^{1}$-embeddding.

Every $x \in(0,1) \backslash \mathbb{Q}$ can be written in the form of the continuous fraction

$$
x=\frac{1}{a_{1}(x)+} \frac{1}{a_{2}(x)+} \frac{1}{a_{3}(x)+} \ldots .
$$

Denote by $A$ the set of all $x \in(0,1) \backslash \mathbb{Q}$ such that

$$
\lim _{B \rightarrow \infty} \limsup _{n \rightarrow \infty} \frac{\sum_{i \leq n, a_{i}(x) \geq B} \log \left(a_{i}(x)+1\right)}{\sum_{i \leq n} \log \left(a_{i}(x)+1\right)}=0 .
$$

The set $A$ is of full Lebesgue measure in $\mathbb{R}$ (see [15], Ch.V.9).

Theorem 15 (Herman). Let $F$ be a $C^{r}$ circle diffeomorphism, $3 \leq r \leq \omega$ and $\rho(F) \in A$. Then $F$ has a unique $C^{r-2}$ embedding.

In paper [1] W. I. Arnold gave the examples of analytic diffeomorphisms which conjugate to its rotations but the conjugating functions are not even absolutely continuous. This means that they have no $C^{1}$-embeddings.

\section{5. $C^{1}$-EMBEDDINGS WITH SEVERAL FIXED POINTS}

If a diffeomorphism of a compact interval has several fixed points then by a simple reasoning we can reduce the problem to the case of two fixed points.

It is a phenomenon that the embedability of diffeomorphisms with two hyperbolic fixed points in $C^{1}$ flow is very exceptional. The problem of finding some criteria for the function to have $C^{1}$ embedding is still open. We know that the set of $C^{1}$-embeddable functions is of the first category in the space of $C^{1}$ diffeomorphisms with two hyperbolic points which have second derivative in fixed points with topology induced by $C^{1}$ norms (see [50]).

S. Karlin and J. McGregor proved that in the class of probability generating meromorphic functions only $C^{1}$-embeddable functions are linear fractional mappings (see [17], [18]).

In wider classes of functions we have only some indirect criteria described by the property of some special functions. 
Theorem 16 ( [42], [43]). Let $f \in \operatorname{Diff}^{2}[0,1], f(x)<x, x \in(0,1)$ and $f$ have two hyperbolic fixed points. If there exists a differentiable solution $g:[0,1] \rightarrow[0,1]$ of Julia functional equation

$$
g(f(x))=f^{\prime}(x) g(x), \quad x \in[0,1]
$$

then $f$ has a unique $C^{1}$-embedding.

A similar result we have also for the convex and absolutely monotonic functions. One can deduce it from A. Smajdor's papers [36] and [37].

It is easy to show that under the assumptions of Theorem 16 there exist the limits

$$
\begin{gathered}
\gamma(x):=\lim _{n \rightarrow \infty} \frac{f^{n}(x)}{\left(f^{n}\right)^{\prime}(x)}, \quad x \in[0,1], \\
\psi_{a}(x):=\lim _{n \rightarrow \infty} \frac{f^{n}(x)}{\left[\left(f^{n}(a)\right)^{k}+\left(f^{n}(x)\right)^{k}\right]^{1 / k}}, \quad x \in[0,1],
\end{gathered}
$$

for every $a \in(0,1)$ and $k>0$. Moreover, $\gamma$ is a $C^{1}$ solution of $(16)$ in $[0,1)$. We have the following criterion of the embeddability.

Theorem 17 ( [21]). If $f \in \operatorname{Diff}^{2}[0,1], \quad f(x)<x, \quad x \in(0,1), \quad s:=f^{\prime}(0)<1, M:=f^{\prime}(1)>1$ and a function $\gamma$ defined by $(17)$ is differentiable at 1 , or $\psi_{a}$ given by $(18)$ is a $C^{1}$ - diffeomorphism for an a $\in(0,1)$ and $k:=-\frac{\log M}{\log s}$, then $f$ is $C^{1}$ embeddable.

If a mapping $f$ is $C^{1}$ embeddable then its embedding is $C^{1}$ conjugate with a group of rational functions called its normal form. This fact let us to give a formula expressing $C^{1}$ embedding. This shows the following result.

For every $0<s<1$ and $k>0$ define

$$
p_{k}^{t}(x):=s^{t} x\left[1+\left(s^{t k}-1\right) x^{k}\right]^{-\frac{1}{k}}, x \in[0,1], t \in \mathbb{R} .
$$

It is easy to verify that the family $\left\{p_{k}^{t}:[0,1] \rightarrow[0,1], t \in \mathbb{R}\right\}$ is a $C^{\infty}$ iteration group.

Theorem 18 ( [21]). Let $f \in \operatorname{Diff}^{2}[0,1], \quad 0<f(x)<x, \quad x \in(0,1), f^{\prime}(0)=: s<1$ and $f^{\prime}(1)=: M>1$ and $k:=-\frac{\log M}{\log s}$. If $\left\{f^{t}: t \in \mathbb{R}\right\}$ is a $C^{1}$-embedding of $f$, then

$$
f^{t}=\psi^{-1} \circ p_{k}^{t} \circ \psi, \quad t \in \mathbb{R}
$$

where $\psi$ is given by (18).

Let us note that the weakening of the regularity of the function $f$ spoils the assertion. In fact, there exist $C^{1}$ iteration groups of $C^{1}$ diffeomorphisms with two hyperbolic fixed points which are not diffeomorphically conjugated to any group $\left\{p_{k}^{t}:[0,1] \rightarrow[0,1], t \in \mathbb{R}\right\}$ (see $[21]$ ).

It turns out that a slight modification of $C^{2}$ mappings in an arbitrary small subinterval in the interior of the domain makes the functions $C^{1}$ embeddable. This property shows the following theorem.

Theorem 19 ( [49]). Let $f \in D i f f^{2}[0,1], \quad 0<f(x)<x, \quad x \in(0,1), \quad f^{\prime}(0) \neq 1, \quad f^{\prime}(1) \neq 1$. Then for every $b \in(0,1)$ and $a \in\left(f^{2}(b), f(b)\right)$ there exists a function $h:[a, b] \rightarrow[f(a), f(b)]$ such that the function

$$
\widetilde{f}(x)= \begin{cases}f(x) & x \in[0,1] \backslash[a, b] \\ h(x) & x \in[a, b]\end{cases}
$$

is $C^{1}$-embeddable. 


\section{Embeddings of COMMUTING HOMEOMORPHisms WITHOUT FIXED POINTS}

If two functions belong to a flow then obviously they commute. We consider the inverse problem: when commuting homeomorphisms belong to the same continuous flow?

Let $I=(a, b)$ and $f, g$ satisfy the following assumption:

(C) $f, g: I \rightarrow I$ are continuous and strictly increasing surjections, $f<i d, g<i d$,

$$
f \circ g=g \circ f
$$

and $f$ and $g$ are iteratively incommensurable that is for every $n, m \in \mathbb{N} f^{n}(x) \neq g^{m}(x), n+m>0, x \in I$.

If we extend the domain of the functions $f$ and $g$ to $[a, b)$, then at the point $a$ they have fixed point. If additionally we assume that $f, g$ are of class $C^{2}, 0<f^{\prime}(a)<1$ and $0<g^{\prime}(a)$, then we get the positive answer to our problem. It is a simple consequence of Szekeres' results presented in the second section. The problem arise when the functions have only the global regularity on the open interval $I$ without any asymptotic property at the end of the interval. It turns out that then even real holomorphic commuting functions have no joint $C^{1}$ embedding. In this case they possess only continuous embeddings.

A fundamental role in the problem of embedding plays the following set

$$
L=\left\{f^{-n} \circ g^{m}(x), n, m \in \mathbb{Z}\right\}^{d} .
$$

$L$ does not depend on $x \in I$ and either $L=c l I$ or $L$ is a Cantor set.

This set lets us to characterize a joint embedding of $f$ and $g$.

Theorem 20 ( $[20])$. Let $f$ and $g$ satisfy $(C) . f$ and $g$ have a joint $C^{0}$-embedding if and only if $L$ is an interval. If $L$ is an interval then there exists a unique $C^{0}$-embedding $\left\{f^{t}: t \in \mathbb{R}\right\}$ of $f$ such that $f^{s}=g$ for an $s \notin \mathbb{Q}$. This embedding is strictly decreasing.

In particular (see [20]) we have

Remark 1. If $f, g \in \operatorname{Diff}^{1}(I)$ and $f^{\prime}, g^{\prime}$ are of bounded variation then $L$ is an interval and consequently $f$ and $g$ are jointly $C^{0}$-embeddable.

If $L$ is not an interval then under suitable assumptions $f$ and $g$ can be embeddable in a flow but nonmeasurable (see [45]).

To avoid the lack of the embeddability or the above mentioned very irregular embedding we extend the notation of flow to the flow of set-valued functions.

Denote $c c[I]:=\{[c, d] \subset I\}$. A family of set-valued functions $\left\{F^{t}: I \rightarrow c c[I], t \in \mathbb{R}\right\}$ such that

$$
F^{u} \circ F^{v}(x)=F^{u+v}(x), \quad u, v \in \mathbb{R}, \quad x \in I
$$

where

$$
F^{u} \circ F^{v}(x):=\bigcup_{y \in F^{v}(x)} F^{u}(y), \quad x \in I
$$

is said to be a set-valued flow. We have the following results.

Theorem 21 ( $[47])$. Let $f$ and $g$ satisfy $(C)$. Then there exists a set-valued flow $\left\{F^{t}: I \rightarrow c c[I], t \in \mathbb{R}\right\}$ such that $f(x) \in F^{1}(x)$ and $g(x) \in F^{s}(x)$ for $x \in I$ and an $s \notin \mathbb{Q}$. Moreover, for every $t \in \mathbb{R}, x \mapsto F^{t}(x)$ is increasing and for every $x \in I, t \mapsto F^{t}(x)$ is strictly decreasing. The values $F^{t}(x)$ are either singletons or closed intervals.

Theorem 22 ( [47]). Let $f$ and $g$ satisfy $(C)$ and $\operatorname{Int} L=\emptyset$. If $f$ possesses an embedding $\left\{f^{t}: t \in \mathbb{R}\right\}$ such that $f^{s}=g$ for an $s \in \mathbb{R}$, then there exists an additive function $\gamma: \mathbb{R} \rightarrow \mathbb{R}$ such that $\operatorname{Im} \gamma$ is countable and

$$
f^{t}(x) \in F^{\gamma(t)}(x), \quad t \in \mathbb{R}, \quad x \in I .
$$


If in hypothesis (C) we drop the assumption of surjectivity then we can consider only the problem of embeddability in a semi-flow. In this case we modify the definition of the limit set $L$ by a restriction of the set of iterative indices to the set $\left\{(n, m) \in \mathbb{N} \times \mathbb{N}: g^{m}[I] \subset f^{n}[I]\right\}$. It turns out that $f$ and $g$ are embeddable in a semiflow if and only if the limit set $L$ is an interval. Then the embedding is unique and continuous. If $L$ is not an interval then $f$ and $g$ are embeddable in a restricted strictly decreasing semiflow that is a family $\left\{f^{t}: I \rightarrow I, t \in T\right\}$, where $T$ is a dense countable subsemigroup of additive semigroup $\left(\mathbb{R}^{+},+\right)$such that $f^{u} \circ f^{v}=f^{u+v}, u, v \in T$. This family is called its $T$-semi-embedding. Moreover, there exists an additive, countable and dense in $\mathbb{R}^{+}$semigroup $G$ such that for each $T$-semi-embedding of $f$ and $g$ we have $T \subset G$. The above results are described in details and proved in papers [20] and [23].

\section{REFERENCES}

[1] V.I. Arnold Small denominators I. On the mapping of a circle into itself, Izv. Acad. Nauk. Math. Serie 25 (1961), $21-86$.

[2] L. Berg, Iterationen von beliebiger Ordung, Z. Angew. Math. Mech. 40 (1960), 215-229.

[3] L.E.J. Brouwer, Beweis des ebenen Translationssatzes, Math. Ann. 72 (1912), no. 1, 37-54.

[4] K. Ciepliński, On the embeddability of a homeomorphism of the unit circle in disjoint iteration groups, Publ. Math. Debrecen 55 (1999), no. 3-4, 363-383.

[5] K. Ciepliński, Some remarks on iteration groups on the circle, Demonstracio Math. 37 (2004), no. 2, 363-373.

[6] R. R. Coifman, Sur l'équivalence des familles d'itération réguliére C. R. Acad. Sci. Paris 259 (1964), 284-286.

[7] R. R. Coifman, Sur l'unicité des solutions de d'Abel-Schröder l'équation et l'itération continue J. Austalian Math. Soc. 5 (1965), 36-47.

[8] R. R. Coifman,Pseudo-groupes de transformations et itration continue des fonctions relles These No. 1393 Imprimerie Albert Kundig, Geneva $196531 \mathrm{pp}$.

[9] I. P. Cornfeld, S. V. Fomin, Ya. G. Sinai, Ergodic theory, Grundlehren der mathematischen Wissenschaften 245, SpringerVerlag, New York 1982.

[10] W. J. Culver, On the existence and uniqueness of the real logarithm of a matrix, Proc. Amer. Math. Soc. 17 (1966), 1146-1151

[11] J. Domsta, A limit formula for regular iteration groups, Aequationes Math. 52 (1996), no. 1-2, 116-134.

[12] S. Dubic, Problémes relatives a l'itération de functions suggérés par les processus en cascade, Ann. Inst. Fourier de l'Univ. Grenoble 31 (1971), 172-251.

[13] M. K. Fort Jr., The embedding of homeomorphisms in flow, Proc. Amer. Math. Soc. 6 (1955), 960-967.

[14] J. Ger, A. Smajdor, Regular iteration in the case of multiplier zero, Aequationes Math. 7 (1972), 65-69.

[15] M. R. Herman, Sur la conjugaison différentiable des difféomorphismes du cercle a des rotations, Inst. Hautes Études Sci. Publ. Math. 49 (1979), 5-233.

[16] E. Hille, R.S. Phillips, Functional analysis and semi-groups, Amer. Math. Soc. Coll. Publ. Vol. 31, Amer. Math. Soc., Providence, RI, 1957.

[17] S. Karlin, J. McGregor, Embeddability of discrete time simple branching processes into continuous time branching processes, Trans. Amer. Math. Soc. 132 (1968), 115-136.

[18] S. Karlin, J. McGregor, Embedding iterates of analytic functions with two fixed points into continuous groups, Trans. Amer. Math. Soc. 132 (1968), 137-145.

[19] Y.Katznelson, D. Ornstein The differentiability of conjugation of certain diffeomorphisms on the circle, Ergod. Th. Dynam. Sys. 9 (1989), 643-680.

[20] D. Krassowska, M. C. Zdun, On the embeddability of commuting continuous injections in iteration semigroups, Publ. Math. Debrecen 75 (2009), no. 1-2, 179-190.

[21] D. Krassowska, M. C. Zdun, On iteration groups of $C^{1}$ diffeomophisms with two fixed points, J. Math. Anal. Appl. 382 (2011), no. 1, 204-213.

[22] D. Krassowska, M. C. Zdun, On the principal iteration semigroups in the case of multipler zero, Cent. Eur. J. Math. 11 (2013), no. 1, 177-197.

[23] D. Krassowska, M. C. Zdun, On one-parameter semigroups generated by commuting continuous injections, J. Math. Anal. Appl., 416 (2014), 862-880.

[24] M. Kuczma, A remark on commutable functions and continuous iterations, Proc. Amer. Math. Soc. 13 (1962), 847-850.

[25] M. Kuczma, On the Schröder equation, Rozpawy Mat. [Dissertationes Math.] 34 (1963), pp 49.

[26] M. Kuczma, Functional equations in a single variable, Monografie Mat. 46, Polish Scientific Publishers, Warszawa 1968.

[27] M. Kuczma, B. Choczewski, R. Ger, Iterative functional equations, Encyclopaedia Math. Appl. 32, Cambridge Univ. Press, Cambridge 1990.

[28] Z. Leśniak, On a decomposition of the plane for a flow free mappings, Publ. Math. Debrecen 75 (2009), no. 1-2, $191-202$.

[29] Z. Leśniak, On homeomophic and diffeomophic solutions of the Abel eqautaion on the plane, Ann. Pol. Math. 58 (1993), no. $1,8-18$. 
[30] Z. Leśniak, On parallelizability of flows of free mappings, Aequationes Math. 71 (2006), no. 3, $280-287$.

[31] Z. Leśniak, On the structure of Brouwer homeomorphisms embeddable in a flow, Abstr. Appl. Anal., vol. 2012 (2012), Article ID 248413, 8 pp.

[32] Z. Leśniak, M. C. Zdun, On iteration groups of singularity-free homeomorphisms of the plane, Ann. Math. Sil. 8 (1994), 203-210.

[33] A. Lundberg, On iterated functions with asyptotic conditions at a fixed points, Arch för Mat. 5 (1963), $193-206$.

[34] H. Michel, Untersuchungen über stetige, monotone Iterationsgruppen reeller Funktionen ohne Differenzierbarkeitsvoraussetzungen, Publ. Math. 9 (1962), 13-46.

[35] A. Smajdor, Regular iterations with multiplier 1, Fund. Math. 59 (1966), 65-69.

[36] A. Smajdor, On some special iteration groups, Fund. Math. 82 (1973), 67-74.

[37] A. Smajdor, Note on the existence of convex iteration groups, Fund. Math. 87 (1975),213-218.

[38] P. Solarz, M. C. Zdun, Embeddings of diffeomorphisms of the plane in regular iteration semigroups Aequationes Math. (2014) DOI. 10.1007/s00010-014-0273-7.

[39] G. Szekeres, Regular iteration of real and complex functions, Acta Math. 100 (1958), 203-258

[40] Gy. Targonski, Topics in iteration theory, Vanderhoeck and Ruprecht, Studia Matematica: Script; 6 (1981).

[41] L.S Yoccoz, Conjugaison differentiable des difféomorphismes du cercle dont le nombre de rotation vérifie une condition Diophante, Ann. Sci. Ec. Norm. Sup. 17, (1984), vvvh 333-361.

[42] M. C. Zdun, Continuous and differentiable iteration semigroups, Prace Nauk. Uniw. Ślask. Katowice. 308, Katowice, 1979.

[43] M. C. Zdun, On differentiable iteration groups, Publ. Math. Debrecen 27 no. 1-2 (1979), $105-114$.

[44] M. C. Zdun, On embedding of homeomorphisms of the circle in continuous flow, Iteration theory and its functional equations (Lochau, 1984), 218-231, Lecture Notes in Math. 1163, Springer, Berlin, 1985.

[45] M. C. Zdun, On the embeddability of commuting functions in a flow, Selected topics in functional equations and iteration theory (Graz, 1991), 201-212, Grazer Math. Ber. 316, Karl-Franzens-Univ. Graz, 1992.

[46] M. C. Zdun, On a limit formula for embeddings of diffeomorphisms in regular iteration semigroups, J. Difference Equations and Application. Vol 19, No 6, (2013),942-951.

[47] M. C. Zdun, On set-valued iteration groups generated by commuting functions, J. Math Anal. Appl. 398 (2013), 638-649.

[48] M. C. Zdun, P. Solarz Recent results on iteration theory: Iteration groups and semigroups in the real case, Aequationes Math., 87 (2014), 201-245.

[49] M. C. Zdun, On approximative embeddability of diffeomorphisms in $C^{1}$-flows, J. Difference Equation and Application, Vol. 20. No 10 (2014), 1427-1436.

[50] Weinian Zhang, A generic property of globally smooth iterative roots, Sci. China Ser. A 38 (1995), no. 3, $267-272$. 